\title{
Sustained Remission After Combination Therapy with Rituximab and Etanercept in Two Patients with Rheumatoid Arthritis After TNF Failure: Case Report
}

\author{
Martin Feuchtenberger*, Christian Kneitz, Petra Roll, Stefan Kleinert and Hans-Peter Tony
}

University of Würzburg, Dept. of Medicine II, Rheumatology and Clinical Immunology, Germany; Department of Medicine II, Klinikum Südstadt Rostock, Germany

\begin{abstract}
Objectives: Approximately up to $40 \%$ of patients with rheumatoid arthritis (RA) fail to respond to tumor necrosis factor (TNF) inhibitors, lose response over time or are unable to tolerate treatment.

Materials and Methodology: We report two female patients suffering from active, refractory rheumatoid arthritis despite TNF blocking agents who have been treated with rituximab added to ongoing therapy with etanercept.

Results: Combination therapy was tolerated without any acute side effects. Both patients improved with a significant, long lasting reduction of disease activity (DAS28, CRP). Evaluation of the immunological parameters showed the expected Bcell depletion and a transient reduction of immunoglobulin-levels. One patient developed four serious infections requiring antibiotic treatment (1 pneumonia, 3 exacerbations of her pre-existing chronic bronchitis) within follow up of 45 months.

Conclusion: Combination therapy of rituximab and etanercept lead to a significant improvement of clinical disease activity and inflammatory parameters in two RA patients refractory to anti-TNF treatment.
\end{abstract}

Keywords: Rheumatoid arthritis, rituximab, etanercept, TNF failure.

\section{INTRODUCTION}

Rheumatoid arthritis (RA) represents a chronic inflammatory disease leading to progressive joint destruction, immobilisation and increased mortality. RA is treated with DMARDs (disease modifying anti-rheumatic drugs) alone or in combination with glucocorticoids and/or so called biologicals, e.g. TNFalpha-antagonists. The introduction of TNFalpha blockers undoubtedly has revolutionized treatment of RA. Nevertheless, up to $40 \%$ of by this means treated patients do not respond adequately, lose response over time or are unable to tolerate treatment requiring alternative therapeutic options. These include a different TNF inhibitor, the T-cell co-stimulation modulator abatacept, or the B-cell depleting rituximab $[1,2]$. Rituximab, a monoclonal antibody that selectively targets CD20-positive B cells, in combination with methotrexate is effective and welltolerated in the treatment of RA patients who have had an inadequate response to one or more TNF inhibitors [3, 4]. It has been discussed recently that efficacy of TNF blocking agents may be restored by combination with rituximab. In general, combination therapy is a well proven concept in RA treatment e.g. combination of conventional DMARDs or methotrexate with TNF blockers, rituximab or abatacept. There are only very few experiences regarding the combination of biologicals. Combination of anti cytokine principles seems to increase susceptibility to infections. Combination of cytokine and cell targeted principles may prove to be

\footnotetext{
*Address correspondence to this author at the University of Würzburg, Dept. of Medicine II, Rheumatology and Clinical Immunology, Klinikstraße 6, D-97070 Würzburg, Germany; Tel: 49-(0)931/201-0; Fax: +49-
} (0)931/201-70421; E-mail: Feuchtenbe_M@klinik.uni-wuerzburg.de advantageous. We report two female patients in whom due to numerous ineffective therapies combination therapy of etanercept and rituximab was applied.

\section{RESULTS}

\section{Patient 1}

62 year old female was diagnosed seropositive RA in 1987. At this time, radiological erosive joint damage was already present. Therapy with oral and later parenteral gold was provided for more than five years with very little clinical benefit. Following this, the patient was successfully treated with methotrexate for four years. MTX had to be terminated in 1999 due to MTX pneumonitis and leflunomide was started. Because of high disease activity and radiological progress (also with involvement of the atlantodental joint) treatment with etanercept was initiated in February 2000 with initially good response. At the end of 2001 an increase in RA activity could be observed and rituximab was applied within the framework of a local pilot study in the use of rituximab for RA February 2002. Altogether 4 infusions of rituximab were administered at weekly intervals in dosages of $375 \mathrm{mg} / \mathrm{m}^{2}$ under premedication with paracetamol $(1000$ $\mathrm{mg}$ ) and clemastin $(1 \mathrm{mg})$ without significant acute side effects. The preceding antirheumatic therapy with etanercept $25 \mathrm{mg}$ twice weekly, leflunomide $20 \mathrm{mg}$ and prednisolone $2.5 \mathrm{mg}$ daily was continued. After rituximab therapy a measurable decrease of disease activity (decline of DAS28 from 5.7 to 4.4 after three months) was observed lasting up to 2007 with a DAS28 < 3 (Table 1). In addition the radiological assessment showed no progression between 2002 and 2006. 25 months after rituximab therapy and 13 months after completed B-cell regeneration, pneumonia was diagnosed 
and treated successfully with antibiotics. In november 2004 (34 months after rituximab), october 2005 (45 months after rituximab) and january 2006 (48 months after rituximab), acute bronchitides as infective exacerbations of the patients known chronic bronchitis occurred and were successfully treated. No opportunistic infection occurred at all. The patient was retreated with rituximab in 2008 due to increasing disease activity with radiological progress compared to 2006. Etanercept was stopped. Five months after retreatment with rituximab clinical remission could be documented (DAS28 1.9).

\section{Patient 2}

A 33 year old female patient was diagnosed a seropositive RA in 1986. Comorbidities in this patient include a successfully treated mammary carcinoma in 1990, osteoporosis and hypercholesteremia. RA treatment so far included Chloroquin (thrombocytopenia), gold parenterally (thrombocytopenia), sulfasalazine (ineffectiveness), methotrexate (mucositis) and leflunomide (ineffectiveness). Etanercept as monotherapy was started in 2000 (2x25 mg/week) with clinical improvement and only minimal residual disease activity. Due to increasing disease activity with destructive arthritis in the left elbow and right wrist joints treatment with rituximab within the framework of a monocentric pilot study was applied in 5/2002 (treatment protocol see above) while therapy with etanercept was continued. The patient improved significantly and the general clinical activity parameters decreased (Table 1). Only a single synovialitis in the left elbow joint persisted, so that local therapy with glucocorticoids as well as consecutive synovectomy of the left elbow joint were done in February 2004. Under continued etanercept treatment, the patient stayed in remission without radiological progress between 2002 and 2005. Relevant infectious complications or opportunistic infections did not occur at any time. Radiological assessment in 2008 revealed a slight progress of erosions in two MTP joints without any change for the hands while clinical remission (DAS28 1.8) persisted under etanercept monotherapy.

\section{Immunological Parameters}

In both patients sufficient $\mathrm{IgG}$ antibody titers against tetanus, mumps, measles, rubella, Epstein-Barr, cytomegalo and herpes virus 1 and 2 prior to commencing rituximab therapy could be detected (data not shown). These antibody titers did not show a falling tendency after rituximab therapy (45 months observation period). Rheumatoid factor (RF) dropped significantly in both patients. In patient 1 , minimal values of 20\% compared to baseline were measured after 15 months. In patient 2 , minimal values of $59 \%$ were reached after 9 months (Table 1). While in patient $1 \mathrm{RF}$ stayed lowered for even 45 months, the rheumatoid factor increased to initial values in patient 2 in the course of time.

In patient 1 immunoglobulins $\mathrm{G}, \mathrm{A}$ and $\mathrm{M}$ levels (Table 1) showed only a transitory drop of IgG levels below the normal range 6 months after RTX therapy. IgM and IgA dropped slightly, but remained within the normal range. Patient 2 showed no relevant variations regarding $\operatorname{IgG}$ and IgA levels.

After therapy a complete depletion of $\mathrm{CD} 19^{+} \mathrm{B}$ cells was observed in both patients (Fig. 1). This lasted for eight months in patient 1 , and 10 months in patient 2. Patient 1 already showed a complete numeric regeneration of CD19 $9^{+}$ B-cells within 12 months after rituximab. In patient 2, the starting level of $\mathrm{CD} 19^{+} \mathrm{B}$-cells was reached after 16 months. During the regeneration phase, the $\mathrm{CD} 27-\mathrm{IgD}^{+}$naive B cells increased continuously (data not shown) and represented the numerically dominant population in the peripheral B-cell pool two years after rituximab. In contrast, $\mathrm{CD} 19^{+} \mathrm{CD} 27^{+}$ memory B cells stayed suppressed (Fig. 1). B cell regeneration was not accompanied by an increase in disease activity.

Table 1. Clinical and Laboratory Parameters After One Cycle of Rituximab (RTX) with Ongoing Etanercept Treatment: DAS28, IgG, IgA, IgM and Rheumatoid Factor (RF); Values Below the Normal Range are Grey (Values in \% Compared to the Starting Level)

\begin{tabular}{|c|c|c|c|c|c|c|}
\hline \multicolumn{7}{|c|}{ Patient 1} \\
\hline Months after RTX & 0 & 3 & 6 & 9 & 15 & 45 \\
\hline IgG $(690-1600 \mathrm{mg} / \mathrm{dl})$ & 884 & $835(94)$ & $671(76)$ & $690(78)$ & 909 (103) & 877 (99) \\
\hline $\operatorname{IgA}(70-370 \mathrm{mg} / \mathrm{dl})$ & 283 & $250(88)$ & $197(70)$ & $210(74)$ & $244(86)$ & $301(106)$ \\
\hline RF (U/l) & 267 & $139(52)$ & $127(48)$ & $62(23)$ & $54(20)$ & $123(46)$ \\
\hline \multicolumn{7}{|c|}{ Patient 2} \\
\hline Months after RTX & 0 & 3 & 6 & 9 & 12 & 45 \\
\hline DAS28 & 5 & 3,9 & 3,3 & 2,58 & 2,44 & 2,78 \\
\hline RF (U/l) & 174 & $131(75)$ & $107(61)$ & $103(59)$ & $136(78)$ & $172(99)$ \\
\hline
\end{tabular}




\section{CD19+ B-cells}

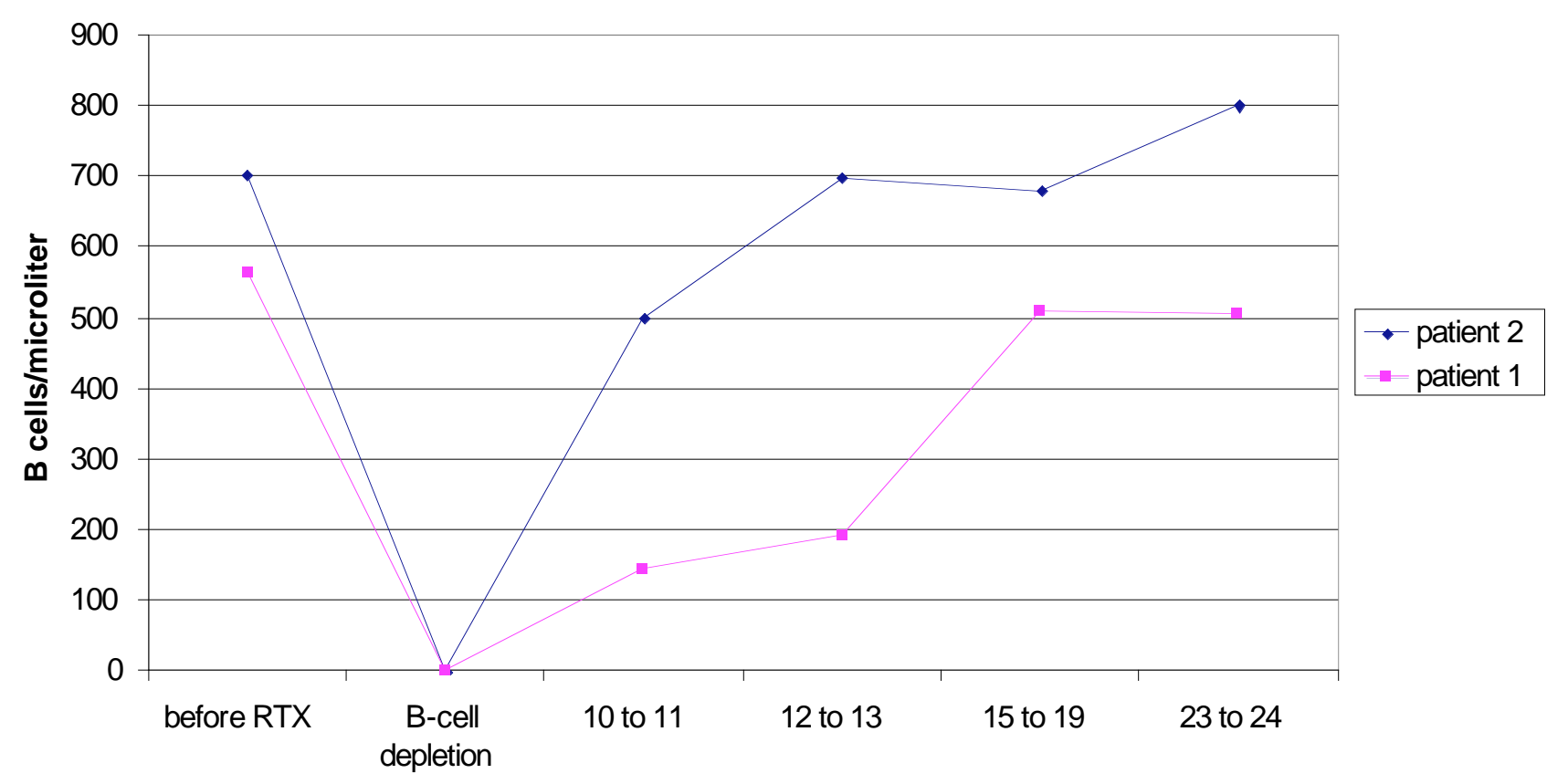

months

CD19+/CD27+ B-cells

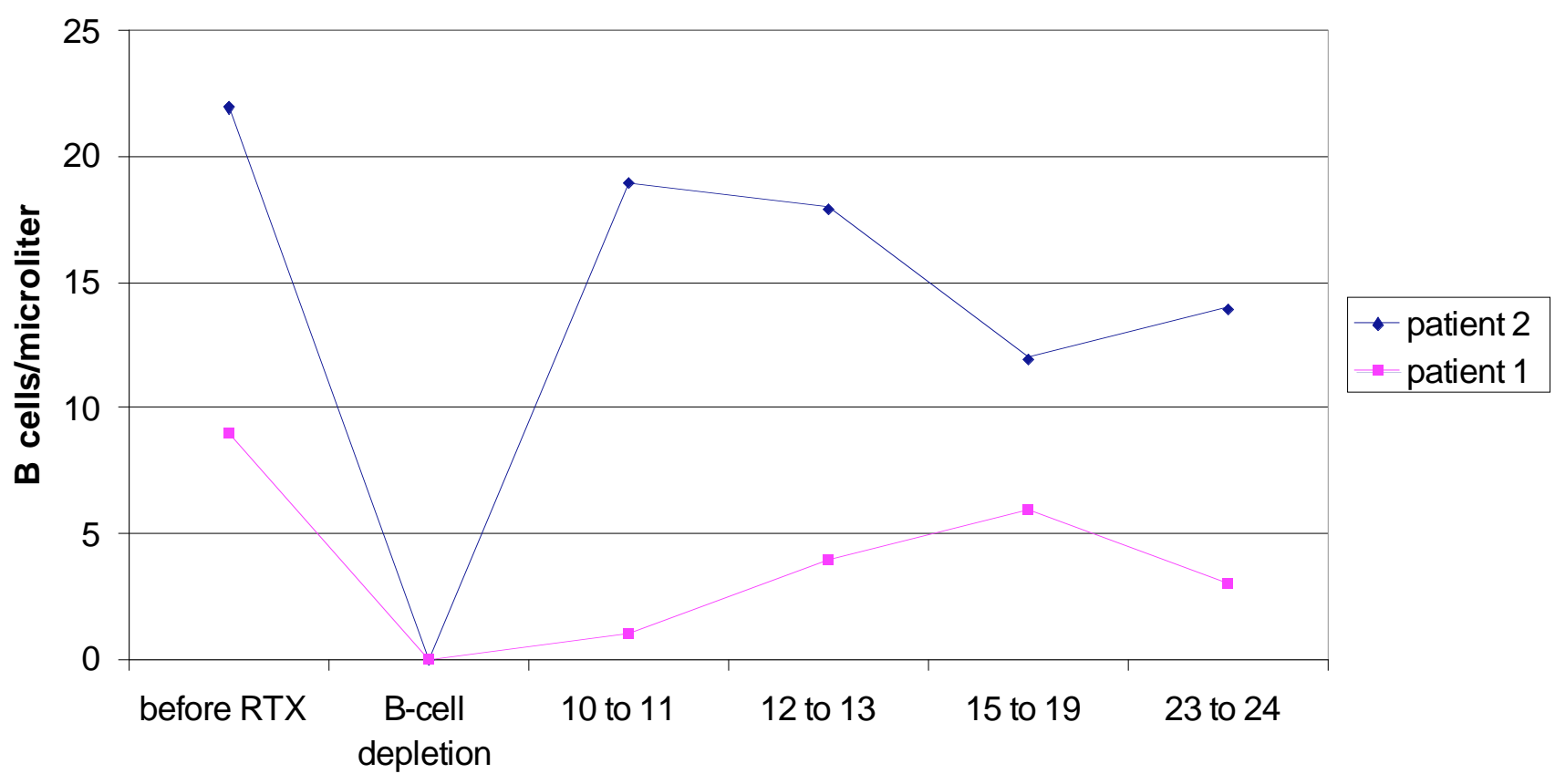

months

Fig. (1). Reconstitution of B cells after one cycle of rituximab (RTX) with ongoing etanercept treatment.

\section{DISCUSSION}

The efficacy of TNF-alpha blockers for rheumatoid arthritis therapy is very well proven in clinical studies and daily praxis. Nevertheless, approximately $40 \%$ of patients with RA treated with TNF inhibitors do not respond well or fail to respond in the course of time. Meanwhile there are some new biological agents having been approved for TNF refractory patients like abatacept or rituximab. Only a limited number of patients treated by this means showed a clinically relevant improvement of disease activity in the situation of 
TNF failure like ACR50 and 70 response [4-7]. Combination therapy therefore may be an option. In general combination therapy is an important and well proven treatment concept in RA and was validated for various substances in clinical studies e.g. combination of conventional DMARDs [8] or combination of methotrexate with TNF-alpha blockers, abatacept or rituximab. On the other hand, there are also data on combination of TNF blocking agents with the costimulation blocker abatacept or anakinra leading to a significant increased infection rate [7,9]. Therefore this combination currently is not recommended.

Combination of a cytokine blocking agent like etanercept and a cell targeted agent like rituximab may have advantages in that respect. The combination of the B-cell depleting antiCD20 antibody rituximab and etanercept in our two patients showed a clinically relevant improvement measured by DAS28, CRP and radiological assessment leading to low disease activity in both patients up to 45 months after initiation of combination therapy. Our data so far are in congruence with data for six patients treated with etanercept and rituximab which have been recently presented (abstract) at the EULAR 2008. A significant reduction in disease activity measured by DAS28 and CRP six months after combination of rituximab and etanercept in these six patients being refractory to etanercept alone was shown, suggesting the superiority of the combination therapy with rituximab [10]. No significant increase in infections and no opportunistic or fatal infections could be observed. Of course, due to the lack of controlled trials, from data available up to now it cannot be proved combination of etanercept and rituximab leading to a synergistic effect in terms of efficacy. Response observed so far could also be attributed to the shift to rituximab per se.

Meanwhile there are also some data available on the safety aspect of a biological combination therapy with rituximab and other biological agents presented at the ACR 2008 (abstract). 185 patients within a safety follow up programme of patients who have been treated at least once with rituximab within an international trial programme were allowed to be treated with another biological agent in the following ( $\mathrm{n}=150$ TNF blocking agents, $\mathrm{n}=25$ abatacept, anakinra or natalizumab) [11]. There was no significant difference in the rate of serious infections after initiation of the second biologic agent (5.49/100pty) compared to the observational period before (6.99/100pty). Serious infections were defined as infections that required intravenous antibiotics or met the regulatory criteria for a serious adverse event like hospitalization, prolongation of an existing hospitalization or immediately life-threatening infections. The median period under review was 186 patient years before and 182 patient years after initiation of the second biological agent. The time of onset (median 7 months after initiation of the second biological agent) or the sort of infections under combination therapy did not show specific characteristics but was more or less typical for RA patients in general (urinary tract infections, pneumonia, bronchitis or gastroenteritis). The infectious complications in our case report (patient 1) were also typical for the patient who was at risk due to her pre-existing chronic pulmonary disease. The infectious complications are unlikely linked to combination therapy of rituximab and etanercept since the onset of infection took place after B cell regeneration was completed and IgG levels have returned to the normal range. Nevertheless, patients with comorbidities like COPD probably may need special surveillance within such concepts.

Both patients showed a significant drop in IgM levels up to $48 \%$ (patient 1) and $24 \%$ (patient 2), respectively, compared to baseline levels without values below the normal range. Such changes are known from larger patient collectives after rituximab therapy and are evidently not linked to heightened risk of infection [3]. In patient 1 , there was a transient reduction in $\mathrm{IgG}$ by more than $20 \%$, which barely lay below the normal range. At the onset of pneumonia, $\operatorname{IgG}$ levels have had normalised for several months. Due to the limited time of $\operatorname{IgG}$ reduction as well as the very slight $\operatorname{IgM}$ reduction months before the infections occurred, a direct relation to the described respiratory tract infections initially appears unlikely. Additionally B cell count and even overall memory B cell count has had normalized for more than 20 months. Regeneration of B-cells in our two patients was mainly carried out by $\mathrm{CD} 27-\operatorname{IgD}$ naive $\mathrm{B}$ while $\mathrm{CD} 19^{+} \mathrm{CD} 27^{+}$memory B cells stayed suppressed. The immunological parameters in our patients with rituximab and etanercept combination therapy did not differ from the data published for rituximab/MTX therapy so far [12].

\section{CONCLUSION}

Our case report shows that the combination of rituximab and etanercept was feasible and lead to a clinically relevant and long lasting improvement in disease activity in two patients with RA refractory to anti-TNF treatment alone. No acute side effects or opportunistic infections could be detected. The combination of rituximab and etanercept therefore maybe a therapeutical option for patients with TNF failure. To address this, there is an obvious need for further studies to secure the casuistically observed good efficacy and compatibility of the TNF blocking agent etanercept with rituximab.

\section{REFERENCES}

[1] Paleolog E. The therapeutic potential of TNF-alpha blockade in rheumatoid arthritis. Expert Opin Investig Drugs 2003; 12(7): 1087-95.

[2] Wolbink GJ, Vis M, Lems W, et al. Development of antiinfliximab antibodies and relationship to clinical response in patients with rheumatoid arthritis. Arthritis Rheum 2006; 54(3): 711-5.

[3] Edwards JC, Szczepanski L, Szechinski J, et al. Efficacy of B-celltargeted therapy with rituximab in patients with rheumatoid arthritis. N Engl J Med 2004; 350(25): 2572-81.

[4] Emery P, Fleischmann R, Filipowicz-Sosnowska A, et al. The efficacy and safety of rituximab in patients with active rheumatoid arthritis despite methotrexate treatment: results of a phase IIB randomized, double-blind, placebo-controlled, dose-ranging trial. Arthritis Rheum 2006; 54(5): 1390-400.

[5] Gomez-Reino JJ, Carmona L. Switching TNF antagonists in patients with chronic arthritis: an observational study of 488 patients over a four-year period. Arthritis Res Ther 2006; 8(1): R29.

[6] Hyrich KL, Lunt M, Watson KD, Symmons DP, Silman AJ. Outcomes after switching from one anti-tumor necrosis factor alpha agent to a second anti-tumor necrosis factor alpha agent in patients with rheumatoid arthritis: results from a large UK national cohort study. Arthritis Rheum 2007; 56(1): 13-20.

[7] Genovese MC, Becker JC, Schiff M, et al. Abatacept for rheumatoid arthritis refractory to tumor necrosis factor alpha inhibition. N Engl J Med 2005; 353(11): 1114-23.

[8] O'Dell JR, Haire CE, Erikson N, et al. Treatment of rheumatoid arthritis with methotrexate alone, sulfasalazine and hydroxychloroquine, or a combination of all three medications. N Engl J Med 1996; 334(20): 1287-91. 
[9] Genovese MC, Cohen S, Moreland L, et al. Combination therapy with etanercept and anakinra in the treatment of patients with rheumatoid arthritis who have been treated unsuccessfully with methotrexate. Arthritis Rheum 2004; 50(5): 1412-9.

[10] Blank N, Max R, Briem S, Lorenz H. Combination Therapy with Rituximab and Etanercept for patients with rheumatoid arthritis. THU0165 EULAR 2008, Paris.
[11] Genovese MC, Breedveld F, Emery P, et al. Safety of Other Biologic Therapies Following Rituximab Treatment in RA Patients. TUE 1671/393, ACR 2008, San Francisco.

[12] Roll P, Palanichamy A, Kneitz C, Dorner T, Tony HP. Regeneration of B cell subsets after transient B cell depletion using antiCD20 antibodies in rheumatoid arthritis. Arthritis Rheum 2006; 54(8): 2377-86.

(C) Feuchtenberger et al.; Licensee Bentham Open.

This is an open access article licensed under the terms of the Creative Commons Attribution Non-Commercial License (http://creativecommons.org/licenses/by$\mathrm{nc} / 3.0 /$ ) which permits unrestricted, non-commercial use, distribution and reproduction in any medium, provided the work is properly cited. 